\title{
Omental Solitary Fibrous Tumor: A Rare Tumor at Rare Site
}

\author{
Abhijeet Ingle ${ }^{1, \odot ~ S u r e n d e r ~ R e d d y ~}{ }^{2} \quad$ Vamshi Krishna Reddy ${ }^{3}$ \\ 1Department of Pathology, Yashoda Hospital, Malakpet, Hyderabad, \\ Telangana, India \\ ${ }^{2}$ Department of General and Laparoscopic Surgery, Yashoda \\ Hospital, Malakpet, Hyderabad, Telangana, India \\ ${ }^{3}$ Department of Medical Oncology, Yashoda Hospital, Malakpet, \\ Hyderabad, Telangana, India \\ Address for correspondence Abhijeet Ingle, MD, Department \\ of Lab Medicine, Yashoda Hospital, Nalgonda X Roads, Malakpet, \\ Hyderabad - 500036, India (e-mail: abhiingle@yahoo.co.in).

\author{
Abstract \\ Keywords \\ - hemangiopericytoma \\ - omentum \\ - primary omental \\ tumors \\ - solitary fibrous tumor
}

Extrapleural solitary fibrous tumor-hemangiopericytoma is a rare tumor. We present a case of this tumor arising in omentum, which is an extremely rare site. The diagnosis was confirmed by diffuse expression of STAT6 on immunohistochemistry. The tumor was assigned a low-risk category according to recent risk categorization models. The patient was advised close follow-up as the tumor was excised completely. These tumors have the potential for recurrence and metastasis even after surgical excision. However, there are no definitive guidelines for adjuvant treatment due to lack of data.

\section{Introduction}

Primary omental tumors are quite rare and include lipomatous tumors, mesothelioma, solitary fibrous tumor-hemangiopericytoma (SFT-HPC), gastrointestinal stromal tumor, leiomyoma, neurofibroma, fibrosarcoma, and leiomyosarcoma. ${ }^{1}$ Omental SFT-HPC is extremely rare, around $10 \%$ of all primary omental tumors. ${ }^{2}$ The term HPC was first coined by Stout and Murray. Enzinger and Smith described the features of these tumors in details. ${ }^{3}$ Now, HPC is regarded as a pattern but not a separate entity and the term preferred is SFT. ${ }^{4}$ We report a case of this rare tumor in omentum with emphasis on immunohistochemistry and recently described risk categorization of these rare tumors.

\section{Case History}

We present a case of 37-year-old female who presented in emergency department with complaints of pain in left lower quadrant of abdomen for the last 15 days with recent exacerbation and nausea. There was no history of loose motions, burning micturition, shortness of breath, or weight loss. She

published online

July 10, 2021
DOI https://doi.org/ $10.1055 / \mathrm{s}-0041-1731942$ ISSN $0974-2727$ was nondiabetic and nonhypertensive. Her menses were regular. On examination, abdomen was soft with tenderness in left lower quadrant. Rest of the general examination was unremarkable.

Computed tomography (CT) scan of abdomen and pelvis showed a lobulated, soft tissue attenuation lesion in left iliac region abutting small bowel loops with significant homogeneous contrast enhancement. There was no evidence of calcifications or bowel wall thickening. Prominent blood vessels were seen adjacent to the lesion. Differential diagnoses offered included neoplastic mesenteric lesion and carcinoid.

Laboratory investigations showed normal hemogram, coagulation profile, renal function tests, and electrolyte levels. She was seronegative for human immunodeficiency virus, hepatitis B surface antigen, and hepatitis $C$ virus.

During exploratory laparotomy, a nodular, brownish, soft to firm mass measuring $6 \mathrm{~cm}$ was seen in the omentum with dilated and congested blood vessels feeding the mass. There was no other pathology in the abdomen. Omentectomy with resection of the mass was performed.

On histopathological examination, the omentum measured $52 \times 18 \mathrm{~cm}$ and showed a nodular, smooth, circumscribed,

C 2021. The Indian Association of Laboratory Physicians.

This is an open access article published by Thieme under the terms of the Creative Commons Attribution-NonDerivative-NonCommercial-License, permitting copying and reproduction so long as the original work is given appropriate credit. Contents may not be used for commercial purposes, or adapted, remixed, transformed or built upon. (https://creativecommons.org/licenses/by-nc-nd/4.0/).

Thieme Medical and Scientific Publishers Pvt. Ltd. A-12, 2nd Floor, Sector 2, Noida-201301 UP, India 
dark brownish mass measuring $6 \times 4 \times 3 \mathrm{~cm}$ with markedly congested and dilated blood vessels in the vicinity ( - Fig. 1). On serial slicing, the mass was grayish brown in color.

Microscopic examination of the mass showed a variably cellular spindle cell tumor with cells arranged in sheets, patternless pattern, and focally in vague storiform pattern with prominent, thin walled, dilated, branching blood vessels (-Fig. 2A). The cells had spindly to plump nuclei with vesicular to granular chromatin, inconspicuous nucleoli, and tapering cytoplasm. Mitotic count was 1 to $2 / 10 \mathrm{hpf}$ in the highest proliferating areas ( $\boldsymbol{- \text { Fig. }}$ 2B). Focal areas of hemorrhages were noted. There were no areas of necrosis or nuclear

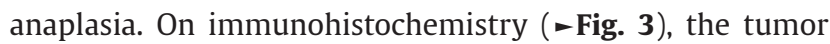
cells diffusely expressed CD34, Bcl2, and STAT6. They were negative for S100, smooth muscle actin (SMA), and c-kit. Ki67 labeling index was around 5\%.

Considering overall features, a diagnosis of SFT with low malignant potential was rendered.

Patient was counseled about the nature of the disease and was advised close follow-up.

\section{Discussion}

SFTs are uncommon mesenchymal neoplasms with ubiquitous location occurring mainly in adults with no sex predilection. Commoner locations are subcutaneous tissue, deep soft tissues of extremities, head and neck especially orbit, thoracic wall, mediastinum, retroperitoneum, and abdominal cavity. Primary omental SFT is extremely rare. ${ }^{5}$ Most of these tumors are circumscribed, well-delineated, and slowly growing painless masses producing symptoms related to mass effects. ${ }^{4}$ The worsening pain in our case may be related to partial torsion of this relatively freely lying tumor as was evidenced by dark brownish discoloration of the tumor and markedly congested, engorged blood vessels in its vicinity. Symptoms related to hypoglycemia are reported in 5\% SFTs mostly in those located in retroperitoneum and pelvis

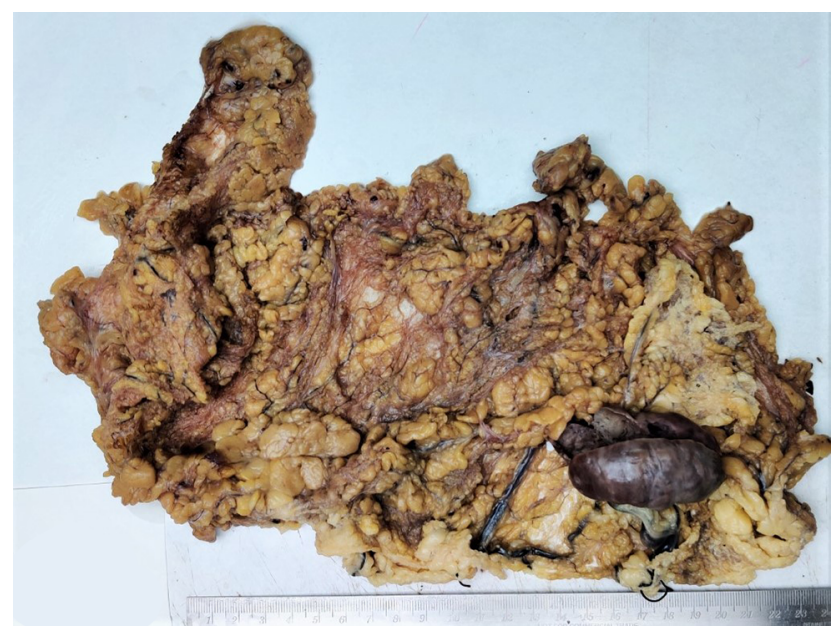

Fig. 1 Gross examination of the omentum showed a nodular, circumscribed, markedly congested, dark brown tumor with dilated and congested blood vessels in the vicinity.

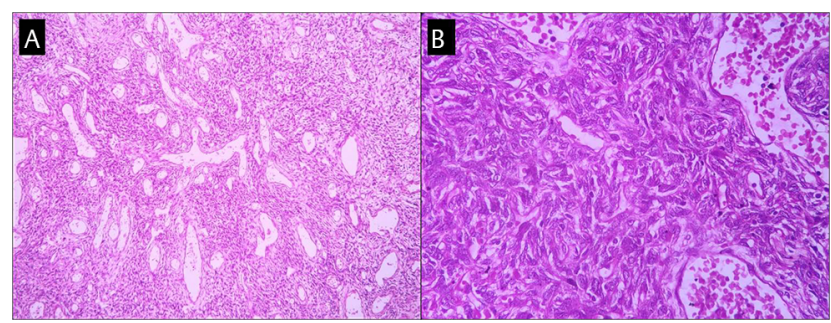

Fig. 2 (A) Tumor cells are spindly, arranged in sheets, pattern less pattern, and vague storiform pattern (Hematoxylin and Eosin stain [H\&E stain], original magnification 100X). (B) Cellular area with cells having spindly to plump nuclei with granular to vesicular chromatin and inconspicuous nucleoli (H\&E stain, original magnification 400X).

through secretion of insulin like growth factors. The symptoms disappear with removal of the tumor. ${ }^{3}$

On CT scan, these tumors are well delineated, isodense to skeletal muscle and show heterogeneous contrast enhancement due to vascularity. Larger, aggressive cases may show increased heterogeneity due to fibrosis, necrosis, hemorrhage, myxoid change, cystic change, or calcifications. ${ }^{4}$ As in our case, imaging may not be able to specify the exact omental location of the tumor.

The tumors grow in deep soft tissues as circumscribed masses or as exophytic mass from serosal surface. On cut section, they are firm, can be nodular, and may show areas of hemorrhages and myxoid change. ${ }^{6,7}$ Rarely, the tumors can involve thoracic and abdominal cavity simultaneously. ${ }^{8}$

On histopathology, the tumors are variably cellular and show haphazardly arranged (patternless pattern) spindle to ovoid cells with vesicular nuclei and dispersed chromatin and indistinct eosinophilic cytoplasm in variably collagenized stroma and thin walled, variably hyalinized, branching (staghorn/hemangiopericytomatous pattern) blood vessels. Also, lipomatous stroma, giant cells, or dedifferentiated areas are described in SFTs. Tumors showing high mitotic activity (>4/10hpf), nuclear atypia, high cellularity, necrosis, infiltrative growth, and size more than $5 \mathrm{~cm}$ are considered malignant. ${ }^{3}$ Recently, a three variable and a modified four variable risk models for the prediction of metastatic risk in SFTs have been proposed and predict their behavior more accurately. Considering the age of patient, mitotic count/10hpf, tumor size, and amount of tumor necrosis, these tumors are stratified into low, intermediate, and high-risk categories. ${ }^{4}$

Mere presence of hemangiopericytomatous vasculature is not diagnostic of SFT as this pattern can be seen in many tumors. Morphological differentials include smooth muscle tumors, neural tumors, spindle cell lipoma, deep fibrous histiocytoma, synovial sarcoma, and dedifferentiated liposarcoma.

On immunohistochemistry, SFTs express Bcl2, CD99, CD34, and can variably express epithelial membrane antigen and S100. Paracentric inversion involving chromosome 12q resulting in fusion of NAB2 and STAT6 genes is pathognomonic for SFT. STAT6 immunohistochemistry is a sensitive and specific surrogate marker for these fusions and helps to rule out its morphological mimics. ${ }^{4}$ However, cases of dedifferentiated liposarcoma showing SFT like areas can be 


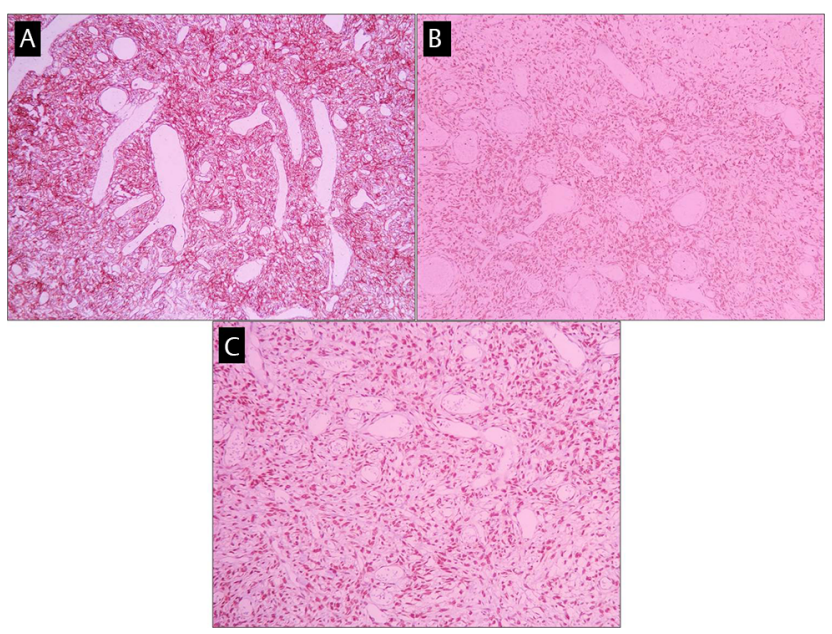

Fig. 3 Tumor cells diffusely express (A) CD34, (B) Bcl2, and (C) STAT6 (immunohistochemistry, 3,3'-diaminobenzidine chromogen, original magnification 100X).

positive for STAT6, although the positivity is more diffuse and stronger in SFT. Presence of varied morphology, liposarcomatous areas, and MDM2 and CDK4 expression on immunohistochemistry or amplification by fluorescence in situ hybridization can help in differential diagnosis. ${ }^{9}$ Malignant cases of SFT can show loss of CD34 expression in higher grade areas along with overexpression of p53 and p16. ${ }^{10}$

Tumor cells in our case expressed CD34 and Bcl2 and were negative for SMA, c-kit, and S100. Expression of STAT6 confirmed the diagnosis of SFT. The tumor, measured $6 \mathrm{~cm}$, was quite cellular at places and showed 1 to 2 mitoses/10 hpf along with focal hemorrhages. Nuclear anaplasia or necrosis was not seen. Hence, it was reported as of low-risk category.

In 15 to $30 \%$ of cases, local or distal recurrences occur and are reported up to 18 years after the surgical excision. Sites of metastasis include lung, liver, brain, scalp, chest wall, gastrointestinal tract, bone, orbit, and lymph nodes. Surgical excision is the main modality of treatment, even in recurrent cases. ${ }^{2}$ Radiation therapy can be given in recurrent or malignant cases. Chemotherapy shows no significant benefit. ${ }^{11}$ In our case, the tumor was excised completely. No adjuvant therapy was advised and the patient was counseled for regular follow-up to rule out recurrence.

Although rare tumor arising at this rare site, SFT should always be considered in the differential diagnosis. Recent availability of STAT6 immunohistochemistry can be used to confirm the diagnosis. The risk stratification models help to predict the behavior of these rare tumors.

\section{Conflicts of Interest}

There are no conflicts of interest.

\section{References}

1 Yoo E, Kim JH, Kim MJ, et al. Greater and lesser omenta: normal anatomy and pathologic processes. Radiographics 2007;27(3):707-720

2 Jaber S, Winer I, Rasool N. Recurrent omental hemangiopericytoma: a therapeutic challenge. Case Rep Obstet Gynecol 2016; 2016:207515710.1155/2016/2075157

3 Weiss S, Goldblum J, eds. Enzinger and Weiss's Soft Tissue Tumors. 5th edition. Philadelphia: Elsevier Inc; 2008 1120-1134

4 Demicco E, Fritche K, Han A, Solitary fibrous tumor. In: Editorial Board. Soft Tissue and Bone Tumors, WHO Classification of Tumors. 5th edition. Lyon: IARC Press; 2020: 104-108

5 Rodriguez Tarrega E, Hidalgo Mora JJ, Paya Amate V, Vega Oomen O. Solitary fibrous tumor of the greater omentum mimicking an ovarian tumor in a young woman. Gynecol Oncol Rep 2016;17:16-19

6 Fukunaga M, Naganuma H, Nikaido T, Harada T, Ushigome S. Extrapleural solitary fibrous tumor: a report of seven cases. Mod Pathol 1997;10(5):443-450

7 Fukunaga $\mathrm{M}$, Naganuma $\mathrm{H}$, Ushigome S, Endo $\mathrm{Y}$, Ishikawa E. Malignant solitary fibrous tumour of the peritoneum. Histopathology 1996;28(5):463-466

8 Maassarani F, Leroy C, Dekeuleneer R. Synchronous abdominal and thoracic solitary fibrous tumour: a case report. Acta Chir Belg 2010;110(2):225-227

9 Doyle LA, Tao D, Mariño-Enríquez A. STAT6 is amplified in a subset of dedifferentiated liposarcoma. Mod Pathol 2014;27(9):1231-1237

10 Mosquera JM, Fletcher CD. Expanding the spectrum of malignant progression in solitary fibrous tumors: a study of 8 cases with a discrete anaplastic component-is this dedifferentiated SFT. ? Am J Surg Pathol 2009;33(9):1314-1321

11 Thway K, Ng W, Noujaim J, Jones RL, Fisher C. The current status of solitary fibrous tumor: diagnostic features, variants, and genetics. Int J Surg Pathol 2016;24(4):281-292 